\title{
ON THE DUST-CURTAIN MODEL OF A RADIATION SHIELD IN A GASEOUS-CORE REACTOR FOR SPACECRAFT
}

\author{
V.C. Liu
Department of Aerospace Engineering \\ University of Michigan \\ Ann Arbor, Mich.

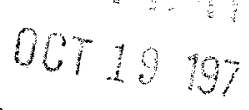

\section{Introduction}

The interest in interplanetary space travel has stimulated much discussion on the desirability of using nuclear, rather than chemical, energy for the spacecraft propulsion. Comparisons of the relative merits of various propulsion systems have been extensively discussed in the literature. The results of such studies yield a diversity of conclusions, depending on the assumptions which are made in such an analysis regarding the rocket parameters, e.g. specific impulse, ratio of the gross weight to the propellant weight and to the propulsion thrust, etc. Suffice it to say, a flight mission, such as an interplanetary travel, would benefit greatly from the use of a high-impulse propulsion, which can best be achieved by means of a nuclear energy source. The principle of rocket propulsion rests on the production of a highly heated working fluid, called the propellant gas, that is expanded and ejected through a nozzle, thereby converting its thermal energy to directed kinetic energy. The heating of the propellant can be derived from chemical reactions of combustibles with a chemical system or nuclear reactions of fissile materials with a nuclear system. In a nuclear reaction the fissile material, such as uranium $\left(U^{235}\right)$ is the fuel and the thermal energy released by fission is transferred to the propellant, usually a gas of low molecular weight, e.g. hydrogen. This energy transfer can be achieved by the use of a solid core, which serves as an intermediate medium for the energy delivery to the propellant. Although the solid-core reactor has reached a prototype engine demonstration after a decade of development, ${ }^{1}$, it does not represent the optimum use of nuclear energy in a rocket engine. This stems from the fact that fission energy is released at very high levels $\left(10 \mathrm{ev}\right.$ or $\left.10^{100} \mathrm{~K}\right)$, which must be degraded in a solid core reactor to the order of $3,000^{\circ} \mathrm{K}$, the limit of the operational temperature of a solid core heat exchanger. It is therefore desirable to use a gaseous core in which the fuel, in the form of highly energetic fission particles, and the propellant gas are intimately mixed, thus transferring energy directly through collision and radiation. The main difficulty in constructing a gaseous core reactor lies in providing a specific flow field for the gaseous mixture in the reactor cavity that fulfills the criticality condition for the nuclear chain reaction and also provides minimum loss of fuel to the exhaust and of energy, in the form of high intensity radiation of neutrons and gamma rays, to the cavity wall. The radiation flux is largely created in the fission process but, in part, comes from absorption processes, the decay of radioactive fission products, and the scattering of neutrons, and is accompanied by some x-rays. These radiations can cause damage to materials, particularly organic materials 
and electric devices. They also cause intensive heating of all the components and materials in the vicinity of the reactor. This points to the importance of an effective radiation shield that minimizes the radiation loss from the nuclear reaction in the cavity.

Various novel concepts of gaseous vortex reactors have been proposed, ${ }^{2}$ e.g. by Kerrebrock and Meghreblian, ${ }^{3}$ suggested that the propellant be ejected at a high tangential velocity into a cylindrical reactor cavity and showed that a peak value of fuel concentration can be maintained at an appropriate radial location with a low concentration of fuel gas near the cylindrical wall and near the vortex center line. This containment of fuel suspension in the flow field is made possible by a dynamic balance between the ordinary diffusion and the pressure diffusion of light and heavy molecular species in the strong pressure gradient of the vortex flow ${ }^{4}$ (FIGURE 1).
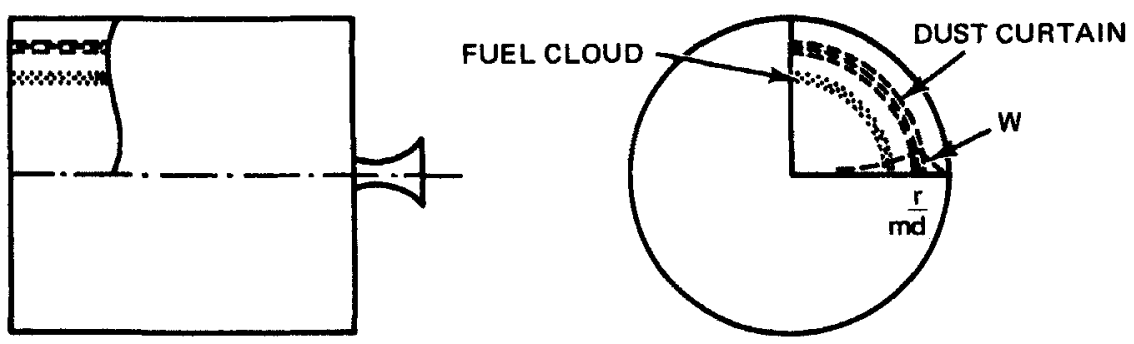

FIGURE 1. Gaseous-vortex reactor: $w=$ distribution of dust $\left(\rho_{\mathrm{d}} / \rho_{\mathrm{p}}\right)$ along radial
istance r.

The concept is that the propellant gas diffuses radially inwards through the hot dense annular cloud of fissioning fuel gas and then flows axially out through an exhaust nozzle at one end of the cylindrical cavity. Such a gaseous-core reactor for rockets has the potential to boost the rocket-specific impulse from a value of 1,000 seconds, an optimum value for a solid-core reactor, to 2,000 seconds and, possibly, more, provided the practical shortcoming of the low rate of hydrogen diffusion through a fuel cloud in a finite-velocity vortex can be removed. Substantial progress has been made in the last decade toward solving complex problems of enclosed vortex flows, heat transfer, heat-resistant materials, and reactor physics pertinent to the vortex reactor model and its modified version. ${ }^{1}{ }^{2}$ The overriding engineering difficulty of the gaseous reactors is the excessive heat loss to the wall, which needs an elaborate cooling system to prevent material failure due to heat load and an unacceptable fuel loss in the exhaust. An obvious difficulty with the gaseous reactors is the specific manner of fuel containment, which is accomplished entirely through fluid dynamic means using no physical barrier. In other words, the strong point of the gaseous reactor, which is the physical simplicity and the direct heat transfer between fuel and propellant, leads to its most serious engineering difficulty. The conceptual innovation of a "dust curtain" as a radiation shield in the cavity of a gaseous core vortex reactor was introduced ${ }^{5}$ for a remedy to the situation. A further advance and study of this new radiation shield is presented here. 


\section{Dust-Seeding Effect}

In an open-cycle, gaseous core engine, e.g. the vortex reactor, there is a steady flow of propellant and fuel gases into and out of the engine cavity which contains a centrally located region of gaseous nuclear fuel (see FIGURE 1). The fission-generated heat is transferred to the propellant through collision and radiation processes. The propellant, e.g. hydrogen, can be made absorptive by the addition some appropriate seeding material, in the form of particles, which is herein called dust gas. Since the temperature of the fissioning core would be in the range of $10,000^{\circ} \mathrm{K}$ to $50,000^{\circ} \mathrm{K}$, most of the thermal radiation from the core would be in the ultraviolet region of the spectrum. In order to determine the characteristics of the radiant heat transfer from the fuel cloud to the propellant, one must know both the spectrum (intensity vs wavelength) of the radiant energy emitted by the fission-generation cloud and the absorption parameter of the seeded propellant as a function of the propellant temperature and radiation wavelength. When radiant energy falls on a cloud of particles suspended in a transparent medium, two processes, absorption and scattering, may occur. It has been shown ${ }^{6}$ that for most seed materials of interest, aerosols of submicron-sized particles absorb much more energy than they scatter. Thus the seeded propellant may generally be treated as a purely absorbing medium. Also, for dispersion of submicron-sized particles in a gas, the particles and gas can generally be considered as in an approximate thermal equilibrium. It is expected that a significant difference in temperature between the dust gas and the propellant occurs only for extremely high heat fluxes.

The absorption of a beam of radiation in a purely absorbing medium is expressed by

$$
I(\lambda, x)=I(\lambda, 0) \exp [-K(\lambda) x]
$$

where $\mathrm{K}(\lambda)$ is the total linear attenuation coefficient at wavelength $\lambda$ and $\mathrm{x}$ is the distance into the medium. For radiation of continuum spectrum, one has

$$
I(x)=\int_{0}^{\infty} I(\lambda, x) d \lambda=\int_{0}^{\infty} I(\lambda, 0) e^{-K(\lambda) x} d \lambda
$$

Thus, if one is given the attenuation coefficient $K(\lambda)$ and the spectrum of the radiation source $\mathrm{I}(\lambda, 0)$, the intensity of the radiation $\mathrm{I}(\mathrm{x})$ at any distance $\mathrm{x}$ into the absorber can be calculated. The energy absorbed in $d x$ is $I(x)-I(x+d x)$. In an actual system the radiation may not be a parallel beam and geometric factors must be taken into account.

If the absorption takes place by more than one process then the total attenuation coefficient equals the sum of the attenuation coefficients for each process separately. The attenuation coefficient of each individual process will depend on the number density and the absorption cross section of the particles in question. In a dust-seeded propellant gas the absorption by both the dust $\left(\mathrm{K}_{\mathrm{d}}\right)$ and propellant $\left(K_{p}\right)$ take place, hence $K(\lambda)=K_{d}(\lambda)+K_{p}(\lambda)$, where $K_{d}$ includes absorption by chemical products produced by the dust as well as by the dust themselves. The attenuation coefficients for submicron-sized particles and 
hydrogen at a wide range of temperature and pressure have been determined by Krascella, ${ }^{6}$ among others.

The attenuation of a fission-particle beam by a dust-propellant mixture due to scattering process can be similarly analyzed, provided the attenuation coefficient of scattering is available. ${ }^{7}$ This additional reflection contributed by the presence of the dust will be found helpful also in maintaining the criticality condition for nuclear chain reaction in the reactor if careful consideration is given to the neutron scattering properties of the dust material being used. The dust reflector serves the purpose of returning (or reflecting) to the core some of the neutrons that would otherwise escape. As a result, the mass of fissile material required to make the core critical is less than in the absence of the dust reflector.

\section{Theory of Floating Dust Curtain}

In the normal operation of a nuclear rocket using dust-seeded propellant, the seeding particles are constantly flushed out of the exhaust nozzle along with the propellant and a minute amount of fuel gas. To achieve a high specific impulse, a rocket parameter defined as the thrust that can be obtained from an equivalent rocket with an exhaust gas flow (weight) rate of unity, it is desirable to have a low mean molecular weight for the exhaust gas of the rocket. The admixture of seeding material that has a molecular weight higher than that of the propellant is a penalty to the rocket specific impulse, hence to the propulsion efficiency. This is particularly significant in interplanetary flight where payload restriction is severe. Ideally one would like to have seeding particles suspended and retained in the reactor cavity to gain the advantage of propellant opacity without paying the price of reduced specific impulse on account of a dust-seeded exhaust. To meet such a requirement, the concept of a dust curtain to be suspended in the vortex reactor, much like the fuel particles, in the form of annular cloud at an appropriate radial distance from the vortex center line was devised. ${ }^{5}$ The dust particles are so granulated in mass and size that their differential centrifugal force and their diffusional drag, both relative to the propellant, are balanced. A bell-shaped distribution (along the radial distance) of dust concentration can be obtained with the concentration peak located between the maximal fuel concentration and the reactor wall. This floating cloud of seeding particles serves the dual purpose of a radiation shield for the wall and a scattering reflector of fission particles. In the following, the mechanics of such a particle suspension scheme will be shown using an idealized model.

Before the fluid dynamics of the dust curtain is formulated, it is necessary to consider the kinetics of small suspended particles in a nonuniform gas. The presence of a second admixture, the fission particles, in the propellant can be justifiably ignored in the present simplified analysis because the maximal concentrations of the two admixtures in the propellant are in separate locations. In accordance with the theory of Waldmann ${ }^{8}$ and also that of Mason and Chapman, ${ }^{9}$ the suspended dust particles are treated as giant molecules. The collisions between the propellant gas molecules (mass $m_{p}$, density $n_{p}$, mass density $\rho_{\mathrm{p}}=\mathrm{m}_{\mathrm{p}} \mathrm{n}_{\mathrm{p}}$ ) and the spherical dust particles (radius $\mathrm{r}_{\mathrm{d}}$, mass $\mathrm{m}_{\mathrm{d}}$, density $n_{d}$ ) are assumed to be elastic, a fraction $\alpha$ rebounding in random 
directions and the remainder $(1-a)$ rebounding specularly. The binary diffusion coefficient $D_{\mathrm{dp}}$ for a quasi-Lorentzian gas can be expressed as follows: 9

$$
\left(\rho_{\mathrm{p}} \mathrm{D}_{\mathrm{dp}}\right)^{-1}=\frac{8 \sqrt{2}}{3}\left(\pi \mathrm{m}_{\mathrm{p}} \mathrm{kT}\right)^{-1 / 2}\left(\pi \mathrm{r}_{\mathrm{d}}{ }_{\mathrm{d}}\right)\left(1+\frac{4}{9} \alpha\right)
$$

assuming $n_{p} \gg n_{d}$ and $m_{d} \gg m_{p}$.

Consider a two-dimensional inviscid vortex flow, without heat source, in a cylindrical cavity. The flow medium is a binary mixture of propellant gas and dust gas which do not react chemically so that each species satisfies a separate continuity equation. Let $\mathrm{n}=\mathrm{n}_{\mathrm{p}}+\mathrm{n}_{\mathrm{d}}, \rho=\rho_{\mathrm{p}}+\rho_{\mathrm{d}}$ denote the mixture number and mass density respectively, $u$ its radial velocity, $r$ the radial distance from the vortex centerline. The continuity equation of the mixture is simply

$$
-2 \pi \text { pur }=\dot{M}_{p}+\dot{M}_{d}
$$

where $M_{p}$ and $M_{d}$ are the mass flows of propellant and dust per unit of vortex length. If $u_{d}$ is the diffusion velocity of the dust gas, its continuity equation becomes

$$
-2 \pi \rho_{\mathrm{d}}\left(\mathrm{u}+\mathrm{u}_{\mathrm{d}}\right) \mathrm{r}=\dot{\mathrm{M}}_{\mathrm{d}}
$$

The binary diffusion equation, with the thermal-diffusion flux ignored, can be written ${ }^{4}$

$u_{2}\left(\frac{\rho}{\rho-\rho_{d}}\right)=-D_{d p}\left[\frac{n}{n-n_{d}} \frac{d}{d r} \ln \left(\frac{n_{d}}{n}\right)+\frac{n\left(m_{p}-m_{d}\right)}{\rho} \frac{d}{d r} \ln p\right]$

where $\mathbf{p}$ denotes the mixture pressure. The two causes producing the diffusion of interest are associated with the two terms on the right of EQ. 6. The first cause is a concentration gradient, and this part of the diffusion flux acts to annul the gradient by random thermal motions tending to render the composition uniform. The second cause is a pressure gradient; this tends to make the heavier particles flow towards the region of greater pressure, as in a cream separator, where the heavier separated milk moves outwards and the lighter cream inwards.

To gain better physical insight, let us rewrite EQ. 6 in terms of the density ratio $\mathrm{w}=\rho_{\mathrm{d}} / \rho_{\mathrm{p}}$ and use the radial momentum equation, $\mathrm{dp} / \mathrm{dr}=\rho \mathrm{v}^{2} / \mathrm{r}$ for a vortex flow, and continuity equations ${ }^{3,5}$

$$
\frac{\dot{\mathrm{M}}_{\mathrm{p}}+\dot{\mathrm{M}}_{\mathrm{d}}}{\dot{\mathrm{M}}_{\mathrm{d}}} \cdot \frac{1+\mathrm{w}}{\mathrm{w}}=\frac{2 \pi \rho_{\mathrm{d}} \mathrm{D} d \mathrm{p}}{\dot{\mathrm{M}}_{\mathrm{d}}}\left(\frac{\mathrm{d} \ln \mathrm{w}}{\mathrm{d} \ln \mathrm{r}} \cdot \frac{\mathrm{m}_{\mathrm{d}}}{\mathrm{m}_{\mathrm{p}}} \frac{\mathrm{v}^{2}}{\mathrm{RT}}\right)
$$


where $R$ denotes the gas constant per unit mass of the propellant, $T$ the propellant temperature. To construct a solution to $\mathrm{EQ}$. 7, the value of the ratio of the radial mass flow rate, $\dot{M}_{d} / \dot{M}_{p}$, at the exit, i.e., the inner boundary where the flow leaves the vortex, must be given. Notice that EQ. 7 is completely analogous to the diffusion equation for the fission particles. ${ }^{3}$ If the dimensionless vortex flow $v^{2} / R T$ is prescribed as a monotonically decreasing function of $r$, a simple maximum for $w(r)$ can be established with $w \rightarrow 0$ as $r \rightarrow \infty$. The position $r_{m d}$ of maximal $w\left(w_{m}\right)$ can be determined by setting $d w / d r=0$ in $E Q .7$.

$$
\frac{\mathrm{v}^{2}}{\mathrm{RT}}=\frac{\Gamma^{2}}{\mathrm{RT} \mathrm{r}^{2}{ }_{\mathrm{md}}}=-\frac{\mathrm{m}_{\mathrm{p}}}{\mathrm{m}_{\mathrm{d}}}\left(\frac{\dot{\mathrm{M}}_{\mathrm{p}}+\dot{\mathrm{M}}_{\mathrm{d}}}{2 \pi \rho_{\mathrm{p}} \mathrm{D}_{\mathrm{dp}}}\right)\left[1 \cdot\left(\frac{1+\mathrm{w}_{\mathrm{m}}}{\mathrm{w}_{\mathrm{m}}}\right)\left(\frac{\dot{\mathrm{M}}_{\mathrm{d}}}{\dot{\mathrm{M}}_{\mathrm{p}}+\dot{\mathrm{M}}_{\mathrm{d}}}\right)\right](8)
$$

A typical bell-shaped curve representing solution w(r) to EQ. 7 is shown in FIGURE 1 . The feasibility is thus established for the containment of dust particles in a vortex flow field under the assumed conditions.

\section{Discussion and Conclusions}

It is admitted that the idealized conditions postulated in the previous section is considerably different from a real gaseous reactor. Two of the major simplifications in the idealized model are the lack of heat sources and of turbulent fluctuations in the assumed vortex flow field. The consequences with the addition of heat sources on the containment of fission particles in a vortex flow have been discussed in the literature. ${ }^{3}$ Their conclusion can be applied to the containment of dust. The effect of turbulence, which probably will be present in a real vortex reactor, on the dust containment is less certain because the extrapolation of the experimental results with laboratory models of unheated vortex may not be valid, because of lack of understanding of turbulent diffusion. It is, however, important to note that the vortex containment of a dust curtain can be made feasible if and when the vortex containment of fission particles becomes an engineering reality because they share the identical problems of fluid dynamics.

It is significant to note from EQ. 8 that the location of the maximal dust concentration $\left(\mathrm{r}_{\mathrm{md}}\right)$ can be prescribed by adjusting the size and mass of the dust particles that are admixed with the propellant. It is also of interest to observe that a dust curtain as such can be introduced in more than one location in a vortex reactor, e.g. on both sides of the maximal fuel concentration, provided two different grades of dust particles are used.

It goes without saying that the present study aims only to discuss the conceptual feasibility of the dust curtain as a radiation shield and a scattering reflector in a vortex reactor. Much work is needed in regard to its engineering feasibility. For instance, the effect of secondary flows into and out of the end-wall boundary layer on the dust containment can be important. 


\section{Summary}

A new concept of radiation shield in a nuclear gaseous reactor for spacecraft is proposed. It entails a cloud of dust particles of specified mass and size that are suspended in a vortex flow of the propellant by fluid dynamic means. The dust ring curtain is suspended between the maximal concentration of the fuel (fission) particles and the reactor cavity wall, thus to serve as a radiation shield for the wall. The mechanics and feasibility of such a dust suspension in a nuclear gaseous reactor, in which the propellant is injected tangentially with a high speed into the cylindrical cavity, are discussed.

\section{References}

1. Cooper, R.S. 1968, Ann. Rev. Nucl. Sci.. 18: 203.

2. McLafferty, G.H. 1967. J. Spacecraft and Rockets 5: 1121.

3. Kerrebrock, J.L. \& R.V. Meghreblian 1961. J. Aerospace Sci. 28: 710.

4. Chapman, S. and Cowling, T.G. 1939. The Mathematical Theory of Nonuniform Gases. Cambridge Univ. Press. London, England.

5. Liu, V.C. 1970. Nature 266: 351.

6. Krascella, N.L. 1966. NASA CR-693.

7. Hasted, J.B. 1964. Physics of Atomic Collisions. Butterworths London, England.

8. Waldmann, L. 1959. Z Naturforsch. 14a: 589.

9. Mason, E.A. S. Chapman 1962. J. Chem. Physics. 36: 627. 